\title{
Spirulina: a new hope for silicosis
}

Keywords: spirulina, silicosis, antioxidant activity, anti inflammatory activity

\section{Opinion}

Silicosis is the permanent scaring of the lungs caused by exposure to crystalline silica, which comes from chipping, cutting, drilling, or grinding soil, sand, granite, or other minerals. Any occupation where the earth's crust is disturbed can cause silicosis. It's commonly found among metal miners, sandstone and granite cutters, foundry workers and potters. The disease is marked by inflammation and scaring of the lungs resulting in nodular lesions in the upper lobes of the lungs. ${ }^{1}$ These scarred areas cannot transfer oxygen into the blood normally. As a result, lungs become less flexible and breathing takes more effort. It is most common form of Pneumoconiosis or Fibrosis and in severe cases causes lung cancer. Symptoms like severe cough, bloodstained sputum, continuous chest pain, and rapid weight loss are prominent leading to disablement and premature death later. Silicosis is an irreversible chronic and progressive disease has no cure and effective treatment. Silicosis cases have been found worldwide. In developing countries situation is at epidemic level, $30-50 \%$ of workers engaged in primary and high risk industries in developing countries may be the victims of the silicosis or other pneumoconiosis.

Although variety of medicinal plants are continuously explored for the cure of various ailments of diseases but there are very few reports on plant based drugs for the treatment of silicosis. As the disease potentially dangerous and having no prominent cure and effective treatment thus researchers must explore the new microorganism or plants based components for the cure of same.

Spirulina, blue green algae can be found the best way to fight durably and effectively against silicosis. Spirulina is well known for its extraordinary nutritional and therapeutic values. It is nature's richest and most complete source of nutrition. Its easy local production with affordable technologies and cost-effectiveness make it valuable for its economic production and easy availability. Spirulina possesses very high amount of antioxidant activities which is due to majorly presence of high amount of phenolic and flavonoids contents in it. A single report by Zongxing et al., ${ }^{2}$ supported effectiveness of Spirulina in induced silicosis rat models. Authors reported elevated level of super oxide dismutase and glutathione in silicosis rats those were fed with Spirulina. The organism has reported anti-inflammatory and analgesic activities. Thus more studies should be conducted on exploration of Spirulina and its various extract for the cure of silicosis. The organism
Volume 8 Issue 2 - 2018

\section{Jaspreet Singh,' Laxmi Parwani \\ 'School of Life Sciences, Jaipur National University, India ${ }^{2}$ Department of Bioscience and Biotechnology, Banasthali Vidyapith, India}

Correspondence: Laxmi Parwani, Assistant Professor, Department of Bioscience and Biotechnology, Banasthali Vidyapith, Banasthali Rajasthan, India, Tel +9I-9982216197, Email laxmi_parwani@rediffmail.com

Received: March 22, 2018 | Published: April 09, 2018

has extraordinary nutritional and therapeutic values, it is safe for human consumption and can be easily cultivated with minimal nutrition requirement and still unexplored for the cure of silicosis. Further detailed and systematic studies in the above mentioned related fields can open hidden treasures of nature in near future.

\section{Conclusion}

Globally, the prevalence of silicosis has risen in recent years. Presently no effective treatment and cure is present for the disease and in scientific world the problem is completely ignored. The present article is providing an effective and suggestive measure for the same. Spirulina can be explored for its potential in the cure of silicosis. As the organism has tremendous nutritional benefits and is rich in antioxidant activities.

\section{Acknowledgement}

Authors declare no acknowledgement.

\section{Conflict of interest}

Authors declare no conflict of interest.

\section{References}

1. WHO. Elimination of silicosis. The global health network. 2007;12:1-18.

2. Zhongxing Z, Li Y, Kaiguo W. Effect of Spirulina on antioxidation of experimental silicosis of rat. Chinese J Pub Health. 2005;21(1):8-9. 